AcCepted to ApJ Letters March 19, 2010

Preprint typeset using LATEX style emulateapj v. 11/10/09

\title{
RADIATIVE TRANSFER MODELS OF A POSSIBLE PLANET IN THE AB AURIGAE DISK
}

\author{
HANNAH JANG-CONDELL ${ }^{1,2,3}$ AND MARC J. KuChNER ${ }^{2}$ \\ Accepted to ApJ Letters March 19, 2010
}

\begin{abstract}
Recent coronagraphic imaging of the AB Aurigae disk has revealed a region of low polarized scattered light suggestive of perturbations from a planet at a radius of $\sim 100 \mathrm{AU}$. We model this darkened region using our fully non-plane-parallel radiative-transfer code combined with a simple hydrostatic equilibirum approximation to self-consistently solve for the structure of the disk surface as seen in scattered light. By comparing the observations to our models, we find that the observations are consistent with the absence of a planet, with an upper limit of 1 Jupiter mass.
\end{abstract}

Subject headings: accretion, accretion disks — circumstellar matter — stars: individual (AB Aur) planetary systems: protoplanetary disks — planetary systems: formation

\section{INTRODUCTION}

The Herbig Ae star, AB Aurigae (AB Aur), hosts a much-studied disk of gas and dust thought to be representative of protoplanetary disks during the Jovian planet formation phase. The circumstellar material around AB Aur has been imaged in scattered light by several observatories, including STIS on HST (Grady et al. 1999), Subaru (Fukagawa et al. 2004), and the Lyot Project (Oppenheimer et al. 2008). These observations show that AB Aur is surrounded by what appears to be a nearly face-on disk, with structures that look like spiral arms.

Using adaptive-optics coronagraphy and polarimetry, Oppenheimer et al. (2008) imaged the AB Aur disk between radii of $43-302$ AU. Their images revealed an azimuthal gap in polarized light at a radius of about 100 AU, along with a $2-\sigma$ bright "spot." They interpreted this to indicate the presence of a possible massive planet of $5-37$ Jupiter masses $\left(M_{J}\right)$.

The position angle (PA) of the putative planet is coincident with the minor axis of the disk, as a well as a gap between two spiral arms observed in other scattered light images (Fukagawa et al. 2004), suggesting that the darkened region results from anisotropies in the overall disk structure, rather than locally confined to a single perturber in the disk. On the other hand, the spiral arms are not seen by Oppenheimer et al. (2008).

Another alternative is that there is no true gap in the disk at all, but rather that the lack of scattered polarized light in this region is purely a geometrical effect caused by the inclination of the disk (Perrin et al. 2009). In this scenario, the disk is inclined enough that the far edge of the disk is back scattering rather than forward sacttering, creating a region of lower total polarized intensity in the back scattering region. This is verified by a lower polarization fraction in the supposed gap, but no corresponding decrease in the total scattered light. This is likely to be the correct interpretation of the AB Aur observation. The objective of this paper is to put firm limits on the mass of any planet embedded in $\mathrm{AB}$

\footnotetext{
${ }^{1}$ Department of Astronomy, University of Maryland, College Park, MD 20742, U.S.A.; hannah@astro.umd.edu

2 NASA Goddard Space Flight Center, Greenbelt, MD 20771

${ }^{3}$ Michelson Fellow
}

Aur, complemetary to the interpretation by Perrin et al. (2009).

Planets embedded in optically thick accretion disks, like the disk around AB Aurigae, are expected to produce perturbations in the density and temperature structure of the disk. Jang-Condell \& Sasselov (2003) and Jang-Condell \& Sasselov (2004) calculated the magnitudes of these perturbations for a range of planet masses and distances. They predicted the formation of a shadow at the position of the planet paired with a brightening just beyond the shadow. Jang-Condell (2008, henceforth Paper I) improved upon these calculations, by self-consistently calculating the temperature and density structures under the assumption of hydrostatic equilibrium and taking the full three-dimensional shape of the disk into account rather than assuming a plane-parallel disk.

Oppenheimer et al. (2008) suggested that the observed structure resembled models of dust trapped in mean motion resonances with a planet (e.g. Kuchner \& Holman 2003). However, AB Aur hosts a gas-dominated disk, as confirmed by detections of numerous molecular species (Mannings \& Sargent 1997, 2000; Dent et al. 2005; Piétu et al. 2005; Corder et al. 2005; Schrever et al. 2008) In such a disk, the dynamics of the gas dominate the system and the dust traces the gas on orbital timescales. Moreover, the disk is optically thick, so the scattered light image traces only features in the upper surface of the disk. Thus, the darkened region and bright "spot" imaged by Oppenheimer et al. (2008) are unlikely to trace dust concentrations, as might be the case were the disk optically thin, but could perhaps be scattering off structure in the disk created in the wake of a planet.

Here we model the observed structure as a shadow caused by the tug of a planet in approximate hydrostatic equilibrium with the gas disk, rather than resonant trapping of ballistic grains. This interpretation is consistent with short stopping time of the dust grains in the gas, $\sim 10^{-3}$ orbits (Youdin \& Chiang 2004). We use the algorithms and code described in Paper I and Jang-Condell (2009, henceforth Paper II) to model the AB Aurigae system (stellar mass $2.4 \pm 0.2 M_{\odot}$, luminosity $48 L \odot$, accretion rate $10^{-7} M_{\odot} \mathrm{yr}^{-1}$; Garcia Lopez et al. 2006; Rodgers 2001). We synthesize images of the disk 
in scattered light and compare them with the coronagraphic images of the AB Aurigae disk to constrain the mass of the perturbing planet.

\section{MODEL DESCRIPTION}

The model we adopt is described in detail in Papers I and II, with parameters adjusted for the AB Aur system. The stellar parameters we adopt are mass $M_{*}=2.4 M_{\odot}$, radius $R_{*}=2.4 R_{\odot}$, and effective temperature $T_{\text {eff }}=10^{4}$ $\mathrm{K}$, which are consistent with $L *=48 L_{\odot}$. For the disk model, we assume a viscosity parameter of $\alpha=0.01$ and accretion rate $\dot{M}=10^{-7} M_{\odot} \mathrm{yr}^{-1}$. Modifying these last two parameters effectively changes only the overall mass of the disk. Since we are only examining the perturbative effects of hypothetical planets in the disk, changing $\alpha$ or $\dot{M}$ do not significantly alter our final conclusions. Indeed, adopting $\dot{M}=10^{-8} M_{\odot} \mathrm{yr}^{-1}$ was not found to alter our overall results.

The amount of heating from stellar irradiation in the disk is sensitive to the opacities used to calculate radiative transfer in the disk. Dust opacities are perhaps the least observationally constrained parameter for disk modeling. Hence, we calculate the opacities using a Mie scattering code developed by Pollack \& Cuzzi (1980). The composition of the dust is that used in Pollack et al. (1994), a mixture of water, troilite, astronomical silicates, and organics. We adopt a size distribution for the dust of of $n(a) \propto a^{-3.5}$ where $a$ is the radius of the grain, with maximum/minimum radii of $1 \mathrm{~mm} / 0.005$ microns. The large maximum grain size represents coagulation of grains in protoplanetary disks. The dust is well-mixed with the gas and uniform in mass opacity throughout the disk. The mean absorption and total extinction (absorption+scattering) averaged over a blackbody of 10,000 K representing the stellar spectrum are $\kappa_{P}^{*}=2.68$ and $\chi_{P}^{*}=17.3 \mathrm{~cm}^{2}-\mathrm{g}^{-1}$, respectively. For radiation at disk temperatures, the relevant opacities are the flux-averaged absorption and Rosseland mean opacity, $\kappa_{R}=2.73$ and $\kappa_{P}=1.46 \mathrm{~cm}^{2}-\mathrm{g}^{-1}$, respectively, which are calculated for a blackbody at $100 \mathrm{~K}$. The temperature at the surface of the disk in the resulting models ranges from 64 to $143 \mathrm{~K}$ over the computation domain, so a choice of $100 \mathrm{~K}$ is a reasonable compromise.

Scattered light dominates the surface brightness of the disk at $1.64 \mu \mathrm{m}$. We calculate scattered light as described in Paper II. The idealized images have been convolved by a Gaussian PSF with FWHM 0.094", the diffractionlimited resolution of the AEOS telescope, to compare to the Lyot Project images of AB Aur (Oppenheimer et al. 2008).

To compare our model to the Oppenheimer et al. (2008) $P$ image in scattered polarized light, we must include the effects of polarization. The size distribution of the dust grains is of $n(a) \propto a^{-3.5}$, so scattering is dominated by the smallest grains. Thus, Rayleigh scattering is a reasonable approximation for the angle-dependent polarization caused by scattering from dust grains (e.g. Graham et al. 2007). The fractional linear polarization from Rayleigh scattering is (Hansen \& Travis 1974)

$$
\begin{aligned}
\frac{P}{I} & =\frac{\left(Q^{2}+U^{2}\right)^{1 / 2}}{I} \\
& =\frac{\sin ^{2} \theta}{1+\cos ^{2} \theta}
\end{aligned}
$$

where $\theta$ is the angle of deflection. We assume that circular polarization is negligible, so $V=0$. Here, $I, Q, U$, and $V$ are the canonical Stokes parameters.

In our model, the scatterers are dust grains in the layer of the disk that is optically thin to stellar light. We assume isotropic single scattering in this optically thin layer of the disk to model the observations. Although our model incorporates thermalization caused by multiple scattering of stellar photons, it does not include the effect of multiple scattering on the polarization of the scattered flux. Monte Carlo calculations of multiple scattering in circumstellar disks (Wood et al. 1996) suggest that multiple scattering can increase the net polarization from a smooth disk by a factor of 1.5 to 2 . But the issue that is important to our study is not the net polarization, it is whether the dimple caused by a planet would be enhanced by multiple scattering effects; we will leave this second-order effect to a future study.

\section{COMPARING THE MODELS TO THE DATA}

The simulated images of the model disk depend on the assumed inclination and orientation. This has been variously reported for $\mathrm{AB}$ Aur as $12-30^{\circ}$ with $\mathrm{PA}$ $60-80^{\circ}$ (Mariñas et al. 2006), $25-35^{\circ}$ with PA $50-60^{\circ}$ (Fukagawa et al. 2004), $23-43^{\circ}$ with PA $58-63^{\circ}$ (Piétu et al. 2005), and $21^{\circ}$ with PA $59^{\circ}$ (Corder et al. 2005). Suffice it to say that the inclination of the disk is highly uncertain, although the PA is largely agreed to be around $60^{\circ}$. We adopt an inclination of $25^{\circ}$ as being a reasonable compromise between the various observations. For simplicity, we assume that the PA of the supposed planet is equal to the PA of the minor axis of the inclined disk.

Fig. 1 shows the real and simulated $P$ images of $\mathrm{AB}$ Aur in scattered polarized light at 1.64 microns, with the observed image from Oppenheimer et al. (2008) in the lower left. We examine disk models with planets of $0,100,300$, and $900 M_{\oplus}(0,0.33,1$, and 3 Jupiter masses), as indicated. The color scale has been calibrated to show the same dynamic range in both the data and the model images. The blacked out inner circle represents the coronograph in the observed image, and the simulation boundary in the model images. The northwest edge of the disk tilted away from the observer.

There is some amount of uncertainty as to the exact location of the star on the observed image, while in the simulated models the stellar position is known exactly. Indeed, Oppenheimer et al. (2008) report an offset in the photocenter of the inner disk of about 88 mas or $12 \mathrm{AU}$ along the minor axis. In order to make a fair comparison between the data and model, we recenter the images by the following procedure.

We select an isophotal contour, with the brightness level set by the bright "spot" in the data image. Although we will henceforth refer to this position as the "planet," we understand it to represent an image feature that may or not correspond to an actual planet. For the model images, we determine the local maximum in brightness in the dimple caused by the 1 Jupiter mass planet, and use this brightness level across all model images. The isophotes are indicated in Fig. 11 by solid black lines. We then calculate the best fit ellipse to this isophote, indicated by the long-dashed white lines. The remaining calculations are now all calculated using the 


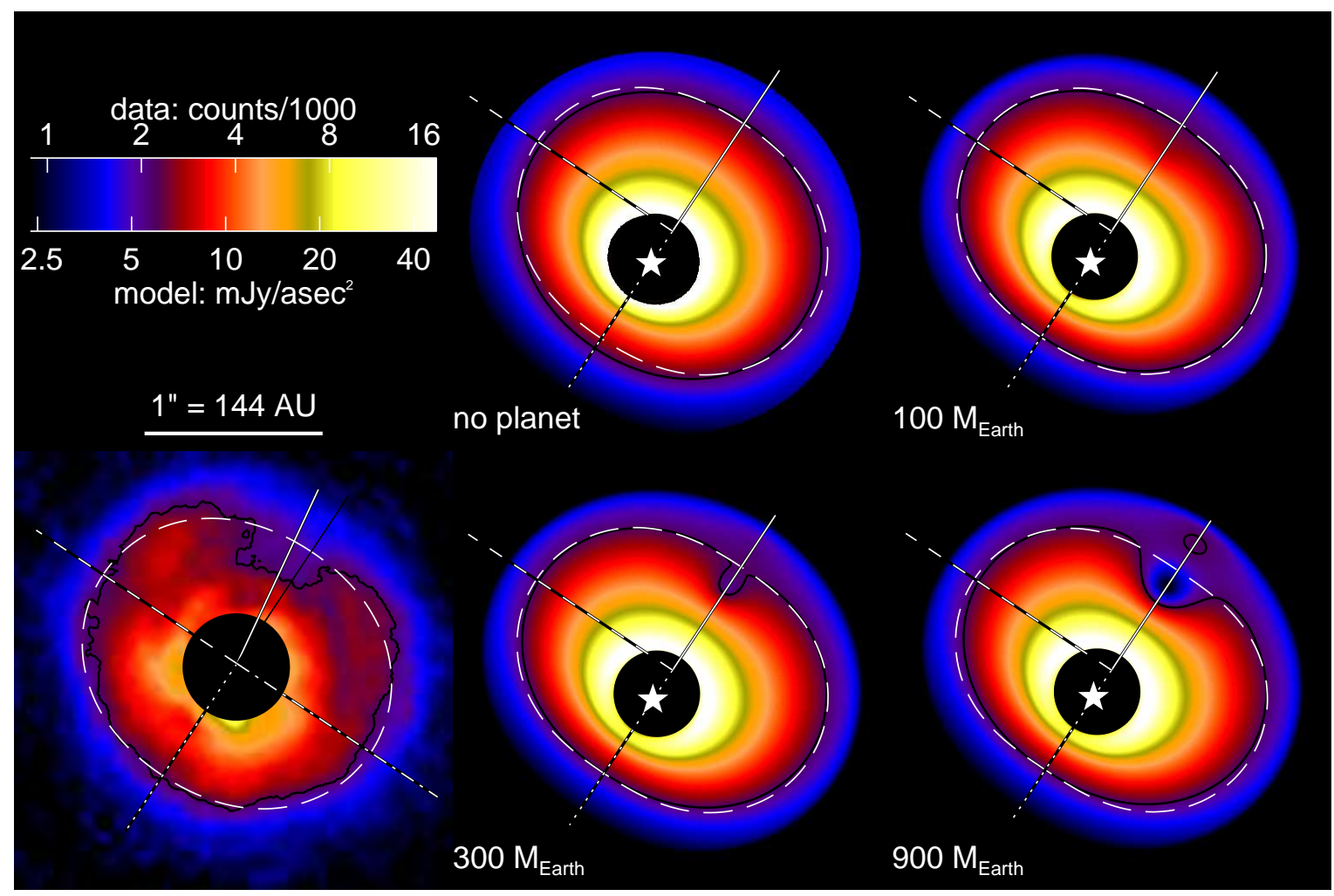

FIG. 1.- Observed $P$ image and simulated images of AB Aur, scaled to the same spatial resolution and dynamic range. The observed image is in the lower left. The blacked out inner circle represents the coronograph in the observed image, and the simulation boundary in the model images. The disk models contain planets of 0 (top center), 100 (top right), 300 (bottom center), and 900 (bottom right) $\mathrm{M}_{\oplus}$. The long-dashed while lines show the best-fitting ellipse to the isophotes indicated by black lines. The solid lines are drawn from the center of the ellipse through the position of the planet, which is equal to the minor axis in the model images. The minor axis is indicated by a black line in the observed image. The dotted white lines show the minor axis opposite the planet. The dashed and dot-dashed lines indicate the major axes of the ellipse.

center of the ellipse as the image center. The calculated $\mathrm{PA}$ for the data is $55^{\circ}$, while the planet's PA is $-25^{\circ}$. The angle between minor axis and planet PA is small, so aligning the planet with the inclination vector of the model disks is a reasonable assumption.

The major and minor axes of the best fitting ellipses for the observed and simulated images are tabulated in Table 1 assuming that $1^{\prime \prime}=144$ AU. The offset listed for the model images is the distance between the star's position and the center of the ellipse. The best-fit ellipses for the model images are slightly more eccentric than that for the data, indicating that either the inclination of $25^{\circ}$ is too high, or that the scattering properties are incompletely modeled. For example, a small amount of forward scattering or slight flattening of the flared disk structure could both create a less eccentric best fit ellipse. However, we are interested in studying the perturbative effect of a planet on the overall disk structure, not creating an exact match to all the disk properties. Indeed, changing parameters such as disk inclination, accretion rate, and disk mass do not significantly change our overall results on the upper limit for the mass of a planet.

In Fig. 1, the white solid, dashed, dotted, and dashdotted lines mark the line through the planet, the northeast major axis, the southeast minor axis, and southwest major axis, respectively. In the simulated images, the planet is aligned with the minor axis, and only one major axis cut is displayed because of symmetry. In the observed image, the minor axis is marked by a black line in the lower left of Fig. 1, offset from the solid white line
TABLE 1

Best-Fit ellipse parameters to isophotal contour, in AU.

\begin{tabular}{lcccc} 
& major axis & minor axis & offset & $\chi_{\nu}^{2}$ \\
\hline data & 129 & 108 & - & - \\
model: & & & & \\
$\quad$ no planet & 131 & 104 & 31 & 0.76 \\
$100 \mathrm{M}_{\oplus}$ & 131 & 103 & 31 & 0.44 \\
$300 \mathrm{M}_{\oplus}$ & 132 & 100 & 29 & 2.18 \\
$900 \mathrm{M}_{\oplus}$ & 133 & 97 & 26 & 16.8
\end{tabular}

through the planet.

In Fig. 2, we plot the surface brightness profiles along the cuts indicated in for the observed and simulated images, with the solid, dotted, dashed, and dot-dashed lines indicating the profiles along the similarly marked cuts in in Fig. 1, with the observed profiles in the lower left, and the remaining plots corresponding to the indicated planet masses. In the observed data, we plot an additional grey line, the profile along the northwest minor axis, indicated by the black line in Fig. 1. This is to show that the profile does not deviate significantly from the profile along the planet line, except for the bright "spot" pointed out in (Oppenheimer et al. 2008). The distances of the major and minor axes of the elliptical fit to the isophotes are indicated by down and up arrows, respectively.

We calculate the error bars according to the following procedure. At a given radial distance from the ellipse center, we sample the brightness along the circle of the given radius. The inclination of the disk introduces an 


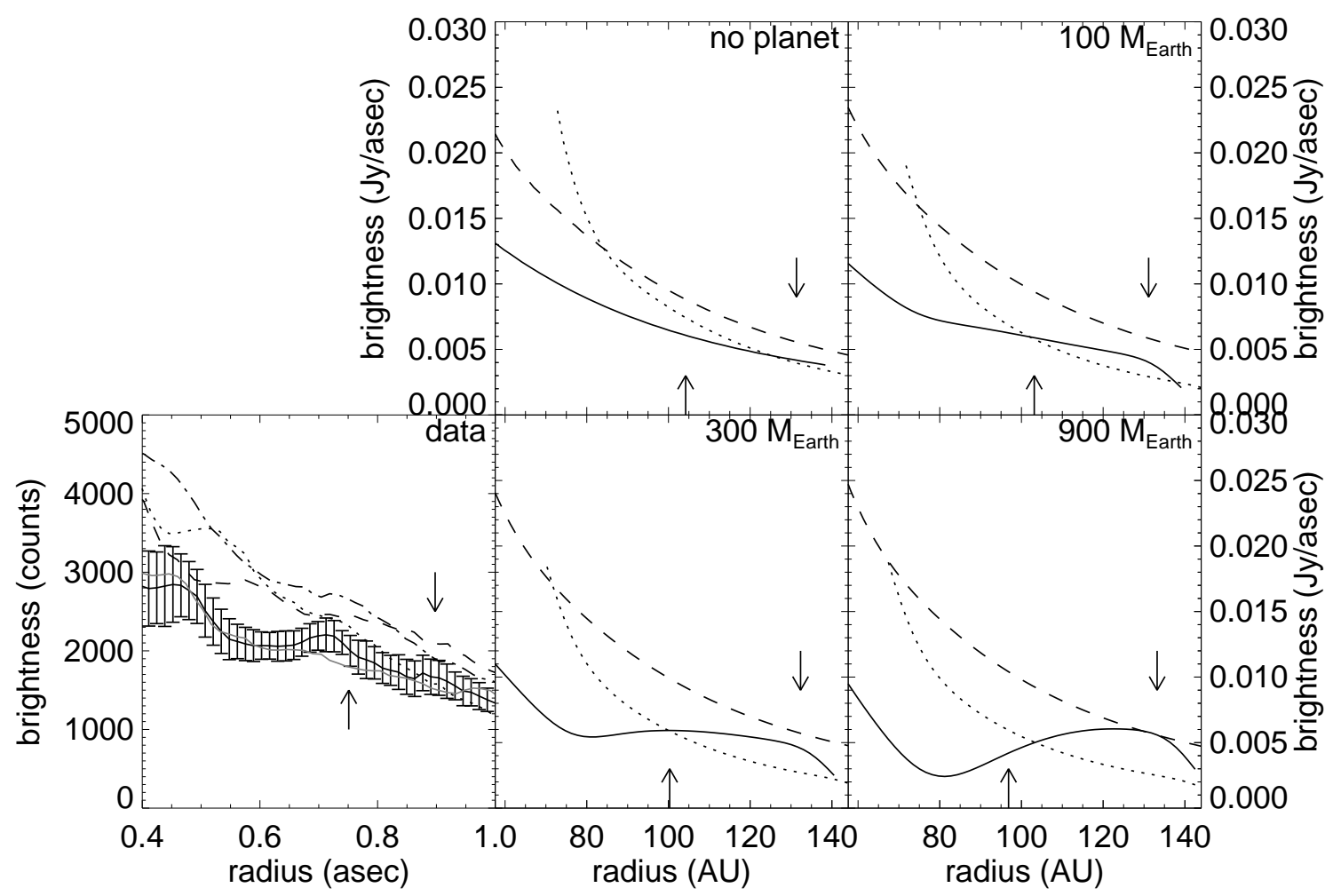

FIG. 2.- Surface brightness profiles along different cuts through the observed and simulated disk images. The observed data are plotted in the lower left, while the remaining plots show the profiles for models with planets of 0 (top center), 100 (top right), 300 (bottom center), and 900 (bottom right) $\mathrm{M}_{\oplus}$. The lengths of the major and minor axes if the isophotal ellipse are indicated by down and up arrows, respectively. The solid lines are the brightness profiles from the center of the ellipse through the position of the planet. The grey line in the lower left plot is the profile along the minor axis of the isophotal ellipse. The dotted lines are profiles along the minor axis opposite the planet. The dashed and dot-dashed lines are profiles along the major axes of the ellipse.

intrinsic variation in brightness that is symmetric about only on axis, so we find the best-fitting function of the form $a+b \sin (\theta+c)+d \sin (2 \theta+e)$ where $\theta$ is the position angle and calculate the rms deviation from this best-fitting function to be the error. The errors are similar in magnitude to those calculated with a best-fitting function of $a+b \sin (\theta+c)$ so we are not over-fitting the data.

The surface brightness profiles of the data (Fig. 2 lower left) are qualitatively simular to those of the planet-less model (Fig. 2 upper middle), with the steepest profile along the southern minor axis (dotted line), and the northern minor axis (solid line) consistently dimmer than the major axes (dashed and dot-dashed). This validates our assumption that the northwestern edge of the disk is tilted away from the observer.

When a planet is added to the image, it creates a dimming in the surface brightness profile slightly inward of the length of the minor axis and a brightening just outward of it. The magnitude of this S-shaped perturbation grows with increasing planet mass. The shifts in the profiles along the other axes can be explained by the shift of the ellipse center as the planet mass increases.

\section{UPPER MASS LIMIT}

In order to establish how well the models match the data, we need to quantify the goodness of the fits. Since we are interested in the perturbations caused by the presence of a planet and only qualitatively interested in the rest of the disk structure, we re-scale the brightness profiles before directly comparing the data to the models.
For a given disk image, we first scale the radius to the length of the minor or major axis, as appropriate, to derive a normalized distance. In the data image, the elliptical radius along the planet's position differs from the minor axis by $1 \%$, so it makes little difference to use the minor axis to scale this profile. We then divide the brightness profile along the planet axis by that of the major axis. This cancels out any small variations that might result from uncertainties in inclination angle. In the case of the data image, we average between the profiles of both major axes before scaling.

The resulting normalized brightness profiles for the data and models are plotted in Fig. 3. Using these profiles and error bars measured from the data, we calculate the reduced $\chi^{2}$ over the normalized radius range from 0.6 to 1.3 . The resulting values of reduced $\chi_{\nu}^{2}$ are tabulated in Table 1. We find that both a Saturn mass planet and no planet at all are good fits to the data, while a Jupiter mass or larger is excluded.

\section{CONCLUSIONS}

We find that scattered polarized light images of $\mathrm{AB}$ Aur do not indicate the presence of a massive planet. We put an upper limit of $1 M_{J}$ on any planet at this location, well below the suggested mass of $5-37 M_{J}$ postulated by Oppenheimer et al. (2008). Indeed, the best models are consistent with the absence of any planet, and support the hypothesis of Perrin et al. (2009), that the "gap" is simply an inclination effect rather than an indication of a planet.

On the other hand, if a massive planet several times 


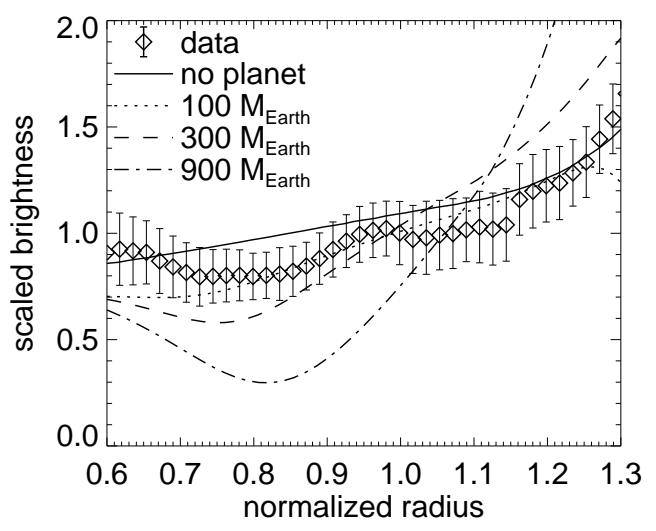

FIG. 3.- Scaled surface brightness profiles through the planet position. The data are plotted as diamonds with error bars. Profiles of disk models with $0,100,300,900 \mathrm{M}_{\oplus}$ planets are shown with solid, dotted, dashed, and dot-dashed lines, respectively.

Jupiter's mass did exist in the disk, our models suggest that it would have been detectable. In other words, our work suggests that massive planets on $100 \mathrm{AU}$ orbits can now be detected via scattered light imaging of nearby Herbig Ae/Be disks.

As a Herbig Ae star, AB Aur will eventually evolve into an A-type main sequence star. We can compare $\mathrm{AB}$ Aur to two main sequence A-type stars with directly imaged planets: Fomalhaut's planet is at 120 AU separation from the star (Kalas et al. 2008), and HR 8799's planets are at 24, 38, and $68 \mathrm{AU}$ separation (Marois et al. 2008). Assuming no significant planetary migration, our upper limits indicate that $\mathrm{AB}$ Aur is not a younger analog of Fomalhaut. The coronographic spot covers the inner 40 $\mathrm{AU}$ of $\mathrm{AB}$ Aur's disk, so it may yet prove to be an ana$\log$ of HR 8799. If planets do migrate significantly after dissipation of the gas disk, and there is some mechanism for expelling planets out to large distances, then AB Aur may evolve into a Formalhaut analog.

If systems like Fomalhaut and HR 8799 are common and planet formation and migration occur early, then there may be many planets waiting to be detected in known Herbig Ae/Be disks. The requirements include high angular resolution $\left(\lesssim 0.1^{\prime \prime}\right)$, good star light suppression, and small inner working angle $\left(\lesssim 0.3^{\prime \prime}\right)$. Ongoing coronographic surveys, like the SEEDS survey on Subaru and future surveys on the Keck, VLT, and Gemini telescopes, will have the power to probe this regime.

The authors are indebted to Ben Oppenheimer for generously providing the observational data needed for this paper. We also thank an anonymous referee for helpful comments that greatly improved our paper. Simulations presented in this paper were carried out using the "borg" cluster administered by the Center for Theory and Computation of the Department of Astronomy at the University of Maryland. Resources supporting this work were also provided by the NASA High-End Computing (HEC) Program through the NASA Center for Computational Sciences (NCCS) at Goddard Space Flight Center. HJC acknowledges support from a Michelson Postdoctoral Fellowship under contract with the Jet Propulsion Laboratory (JPL). JPL is managed for NASA by the California Institute of Technology.

\section{REFERENCES}

Corder, S., Eisner, J., \& Sargent, A. 2005, ApJ, 622, L133

Dent, W. R. F., Greaves, J. S., \& Coulson, I. M. 2005, MNRAS, 359,663

Fukagawa, M., Hayashi, M., Tamura, M., Itoh, Y., Hayashi, S. S., Oasa, Y., Takeuchi, T., Morino, J.-i., Murakawa, K., Oya, S., Yamashita, T., Suto, H., Mayama, S., Naoi, T., Ishii, M., Pyo, T.-S., Nishikawa, T., Takato, N., Usuda, T., Ando, H., Iye, M., Miyama, S. M., \& Kaifu, N. 2004, ApJ, 605, L53

Garcia Lopez, R., Natta, A., Testi, L., \& Habart, E. 2006, A\&A, 459,837

Grady, C. A., Woodgate, B., Bruhweiler, F. C., Boggess, A., Plait, P., Lindler, D. J., Clampin, M., \& Kalas, P. 1999, ApJ, 523, L151

Graham, J. R., Kalas, P. G., \& Matthews, B. C. 2007, ApJ, 654, 595

Hansen, J. E. \& Travis, L. D. 1974, Space Science Reviews, 16, 527

Jang-Condell, H. 2008, ApJ, 679, 797

-. 2009, ApJ, 700, 820

Jang-Condell, H. \& Sasselov, D. D. 2003, ApJ, 593, 1116

-. 2004, ApJ, 608, 497

Kalas, P., Graham, J. R., Chiang, E., Fitzgerald, M. P., Clampin, M., Kite, E. S., Stapelfeldt, K., Marois, C., \& Krist, J. 2008, Science, 322, 1345

Kuchner, M. J. \& Holman, M. J. 2003, ApJ, 588, 1110
Mannings, V. \& Sargent, A. I. 1997, ApJ, 490, 792

-. 2000, ApJ, 529, 391

Mariñas, N., Telesco, C. M., Fisher, R. S., Packham, C., \& Radomski, J. T. 2006, ApJ, 653, 1353

Marois, C., Macintosh, B., Barman, T., Zuckerman, B., Song, I., Patience, J., Lafrenière, D., \& Doyon, R. 2008, Science, 322, 1348

Oppenheimer, B. R., Brenner, D., Hinkley, S., Zimmerman, N., Sivaramakrishnan, A., Soummer, R., Kuhn, J., Graham, J. R. Perrin, M., Lloyd, J. P., Roberts, Jr., L. C., \& Harrington, D. M. 2008, ApJ, 679, 1574

Perrin, M. D., Schneider, G., Duchene, G., Pinte, C., Grady, C. A., Wisniewski, J. P., \& Hines, D. C. 2009, ApJ, 707, L132

Piétu, V., Guilloteau, S., \& Dutrey, A. 2005, A\&A, 443, 945

Pollack, J. B. \& Cuzzi, J. N. 1980, Journal of Atmospheric Sciences, 37, 868

Pollack, J. B., Hollenbach, D., Beckwith, S., Simonelli, D. P., Roush, T., \& Fong, W. 1994, ApJ, 421, 615

Rodgers, B. M. 2001, PhD thesis, University of Washington

Schreyer, K., Guilloteau, S., Semenov, D., Bacmann, A.

Chapillon, E., Dutrey, A., Gueth, F., Henning, T., Hersant, F., Launhardt, R., Pety, J., \& Piétu, V. 2008, A\&A, 491, 821

Wood, K., Bjorkman, J. E., Whitney, B., \& Code, A. 1996, ApJ, 461,847

Youdin, A. N. \& Chiang, E. I. 2004, ApJ, 601, 1109 